Honam Mathematical J. 33 (2011), No. 4, pp. 617-628

http://dx.doi.org/10.5831/HMJ.2011.33.4.617

\title{
ARRANGEMENT OF ELEMENTS OF LOCALLY FINITE TOPOLOGICAL SPACES UP TO AN ALF-HOMEOMORPHISM
}

\author{
SAng-Eon Han And Chun, WoO-JIK
}

\begin{abstract}
In relation to the classification of finite topological spaces the paper [17] studied various properties of finite topological spaces. Indeed, the study of future internet system can be very related to that of locally finite topological spaces with some order structures such as preorder, partial order, pretopology, Alexandroff topological structure and so forth. The paper generalizes the results from [17] so that the paper can enlarge topological and homotopic properties suggested in the category of finite topological spaces into those in the category of locally finite topological spaces including ALF spaces.
\end{abstract}

\section{Introduction}

It is well known that Alexandroff spaces can be considered to be a generalization of finite topological spaces. Further, locally finite spaces also can be viewed as a generalization of finite spaces. Recently, the study of locally finite spaces has been strongly used in the field of applied topology such as digital geometry, digital topology and network science. The paper [17] also studied topological and homotopic properties of finite spaces.

A locally finite space (briefly, LFS) can be locally represented in a computer, which enables one to perform topological investigations, experiments and calculations by means of computers. The concept of

Received October 11, 2011. Accepted December 1, 2011.

2000 Mathematics Subject Classification. 54A10, 54C05, 55R15, 54C08, 54F65, $68 \mathrm{U} 05,68 \mathrm{U} 10$.

Key words and phrases. Locally finite topological space, ALF space, homeomorphism, digital topology, Khalimsky topology, network.

This work was supported by the IT R\&D program of KCC/KCA" [KCA-2011(11913-05001), Development of Future Internet common conceptual architecture and inter-operation techniques for trustworthy communication]. 
LFS is also related to Khalimsky spaces. Compared with a Khalimsky topological space, an LFS is a more general one because an element of a general LFS can have an arbitrary shape, e.g. that of a triangle, a disc, a sphere, a rectangular, a trapezoid and so forth (see Figure 1).

The works $[11,12]$ suggested an axiomatic approach to the theory of LFS. An LFS satisfying these axioms will be called an axiomatic locally finite (for short, ALF) space. Due to the several axioms for being an ALF space, we can efficiently study locally finite spaces. All related notions are described in [11].

The paper is built on the papers $[8,11,13,17]$. In particular, the paper [17] studied various topological and homotopic properties of finite spaces. The aim of the paper is to generalize the result of [17] related to the study of finite spaces into that of locally finite topological spaces so that we can have a very useful tool handling locally finite spaces in terms of a homeomorphism in the category of locally finite spaces including ALF spaces,

\section{Some Properties of ALF Spaces}

The paper [13] (see also [9]) has presented some explanations of the axioms of ALF spaces with the purpose of making them more comprehensible. We repeat here some of these explanations together with some definitions and examples of [13](see also [9]) to make the present paper self-contained. The axioms mentioned before use the notion of a special kind of smallest neighborhood which needs not to be a classical topological neighborhood. The notion of a neighborhood was introduced there axiomatically in a way characteristic for the so-called neighborhood spaces described for example in the classical textbook by Seifert and Threlfall [15]. Following the classical textbook by Seifert and Threlfall [15], we consider a neighborhood space or simply a space as $S=(E, U)$, where $E$ is a nonempty set and $U$ is a system of subsets of $E$, with the property that each element $e$ of $E$ is contained in some element of $U$, and that each such set belonging to $U$ and containing $e$ is called a neighborhood of $e$. A space is called locally finite (briefly, $\mathrm{LF}$ ) if each element of $E$ has a finite neighborhood. In what follows we consider a particular case of LF space satisfying the following Axioms 1 to 4 which we shall call an ALF-space.

Axiom 1. [13] (see also [9]) For each element $e$ of the space $S$, the intersection of any two neighborhoods of $e$ is again a neighborhood of $e$, that is, whenever $A, B \in U$ and $e \in A \cap B$ then $A \cap B \in U$. 
Due to Axiom 1, we can obtain the smallest neighborhood of $e$ that is the intersection of all neighborhoods of $e$. Thus each neighborhood of $e$ contains its smallest neighborhood. Let $\mathbf{Z}$ be the set of integers. For $\{a, b\} \subset \mathbf{Z}$ with $a \leq b$, we use the notation $[a, b]_{\mathbf{Z}}:=\{n \in \mathbf{Z} \mid a \leq n \leq b\}$. We shall denote the smallest neighborhood of $e$ by $S N(e)$.

Example 2.1. In Figure 1(a) we can consider smallest open neighborhoods of elements as follows: Assume that the space $X$ consists of eight elements, i.e. four faces named by $g_{i}, i \in[0,3]_{\mathbf{Z}}$ and four line segments named by $d_{i}, i \in[0,3]_{\mathbf{Z}}$. Then we can consider the following: $S N\left(d_{0}\right)=\left\{d_{0}, g_{0}, g_{1}\right\}, S N\left(g_{i}\right)=\left\{g_{i} \mid i \in[0,3]_{\mathbf{z}}\right\}$. Consider the object $X^{\prime}:=\left\{c_{i}^{0}, c_{i}^{1} \mid i \in[1,4]_{\mathbf{Z}}\right\}$. Then $S N\left(c_{i}^{0}\right)=\left\{c_{i}^{1}, c_{i}^{0}, c_{i(\bmod 4)+1}^{1}\right\}$, and for each $c_{i}^{1}, i \in[1,4]_{\mathbf{Z}}$ we obtain that $S N\left(c_{i}^{1}\right)=\left\{c_{i}^{1}\right\}$.

It will be demonstrated later on that the smallest neighborhoods of an ALF space are open subsets of $S$ in the classical sense (see Corollary $2.5)$.

Axiom 2. [13] (see also [9]) There are space elements which have in their $S N$ more than one element.

In order to establish further Axioms 3 and 4, we need to recall the following basic notions.

Definition 1. [11] If $b \in S N(a)$ or $a \in S N(b)$, then the elements $a$ and $b$ are called incident to each other.

The notion of incident elements seems perhaps to be similar to the adjacency introduced in [14] (for more details on multi-dimensional case, see $[4,6,7])$. There is, however, an important difference between them because we do not suppose that all elements have the same number of incident elements.

Definition 2. [13] Let $S=(E, U)$ be an $L F$ space and $A$ a subset of the set $E$. A sequence $\left(a_{1}, a_{2}, \cdots, a_{k}\right), a_{i} \in A(i=1,2, \cdots, k)$ in which each two subsequent elements are incident to each other, is called an incidence path in $A$ from $a_{1}$ to $a_{k}$.

Definition 3. [11] Incident elements are called directly connected. $A$ subset $A$ of the space $S$ is connected if and only if for any two elements of $A$ an incidence path exists containing these two elements, which completely lies in $A$.

It has been demonstrated in [12] (page 34) that in the case of ALF spaces the connectivity based on the incidence is equivalent to the topological connectivity. 
The author of $[11,12]$ next formulates axioms related to the notion of a boundary. The definition of the topological boundary or of the frontier is as follows:

Definition 4. [13] (see also [9]) Let $S=(E, T)$ be a topological space. The topological boundary, also called the frontier, of a nonempty subset $A$ of the set $E$ is the set of all elements $e$ of $E$ such that each neighborhood of e contains elements of both $A$ and its complement $E \backslash A$.

We shall denote the frontier of $A \subset E$ by $\operatorname{Fr}(A, S)$ and call it the frontier of $A$ in $S$.

The next two axioms are related to the notion of the frontier. In Definition 4 a neighborhood $V$ of an element $e \in E$ is understood as a subset of $E$ containing a subset $O \subset V$ containing $e$ which is open in the space $S$ in the classical sense. Then the author of $[11,12]$ introduces the notions of the neighborhood relation, opponents and those of thick and thin frontiers.

Definition 5. [13] For any $L F$ space $S=(E, U)$ with the smallest neighborhoods $S N: E \rightarrow 2^{E}$, the neighborhood relation $N$, defined as follows: The ordered pair $(a, b)$ is in $N$ if and only if $a \in S N(b)$.

Definition 6. [11] A pair $(a, b)$ of elements of the frontier $\operatorname{Fr}(A, S)$ of a subset $A \subset E$ are opponents of each other if a belongs to $S N(b), b$ belongs to $S N(a)$, one of them belongs to $A$ and the other one to the complement $E \backslash A$.

Definition 7. [11] The frontier $\operatorname{Fr}(A, S)$ of a subset $A$ of a space $S$ is called thick if it contains at least one pair of opponents. Otherwise the frontier is called thin.

To justify of the notation "thick" let us remark that at locations where there are opponent pairs in the frontier, the frontier is doubled: There are two subsets of the frontier which run "parallel" to each other.

Axiom 3. [11] The frontier $\operatorname{Fr}(A, S)$ of any subset $A \subset S$ is thin.

According to Definition 4, the frontier of $A$ is the same as the frontier of its complement $E-A$.

Theorem 2.2. [11, 12] A locally finite space $S$ satisfies the Axiom 3 if and only if the neighborhood relation $N$ of the space $S$ is antisymmetric.

Axiom 4. [11] The frontier of $\operatorname{Fr}(A, S)$ is the same as $\operatorname{Fr}(A, S)$, i.e. $\operatorname{Fr}(\operatorname{Fr}(A, S), S)=\operatorname{Fr}(A, S)$. 
In order to formulate the topological structure of an ALF space, the author of $[11,12]$ has introduced the notion of open and closed subsets of an ALF space $S$ in the way characteristic for neighborhood spaces (compare [15], p. 24).

Definition 8. [11] Let $S=(E, U)$ be an $A L F$ space. A subset $O \subset E$ is called open in $S$ if it contains no elements of its frontier $\operatorname{Fr}(O, S)$. A subset $C \subset E$ is called closed in $S$ if it contains all elements of $\operatorname{Fr}(C, S)$.

Lemma 2.3. [11] Let $S=(E, U)$ be an ALF space with the smallest neighborhoods $S N: E \rightarrow 2^{E}$. A subset $A \subset E$ is open in $S$ according to Definition 8 if and only if it contains together with each element $e \in A$ also its smallest neighborhood $S N(e)$.

The following theorem proves by using Axiom 1 and Definitions 4 and 8 that an ALF space satisfies the classical axioms of topology.

Theorem 2.4. [11, 12] The family of subsets of an ALF space $S$ which are open in $S$ according to Definition 8 forms a topology on $S$.

Corollary 2.5. [11, 12] The smallest neighborhood of any element $e$ of an ALF space $S$ is open both according to Definition 8 and in the classical sense. It is the smallest open subset of $S$ containing the element e.

It is possible to state on the base of Corollary 2.5 that the smallest neighborhoods of all elements of an ALF space $S$ compose a base of the equivalent topological space. All properties of subsets of $S$ can be expressed in terms of the smallest neighborhoods. The definition of an ALF space may be then formulated as follows:

Definition 9. [13] An axiomatic locally finite space (or ALF space for short) is a pair $S=(E, N)$ where $E$ is any set and $N$ is a partially ordered and binary relation on $E$. The relation $N$ specifies for each $e \in E$ its smallest neighborhood $S N(e)=\{x:(x, e) \in N\}$. The subset $S N(e)$ is called the smallest neighborhood of $e$. The space $S$ must satisfy the Axioms 1 to 4 presented above.

Each ALF space is a certain kind of topological space, determined by the family of smallest neighborhoods. In [12] it is proved that the result is a $T_{0}$ Alexandroff space. 


\section{Homeomorphism between ALF spaces}

Since the notion of homeomorphism between two topological spaces is an essential concept of topology, the recent paper [9] studied its properties in the category of ALF spaces in terms of a continuous map in the category of ALF spaces. Besides, the papers [5, 6, 8] also developed several kinds of homeomorphisms and isomorphisms for studying digital spaces. Let us now recall the notion of continuous map in the category of ALF spaces, as follows:

Definition 10. [9] Let $X$ and $Y$ be two ALF spaces. We say that a map $F: X \rightarrow Y$ is continuous on $X$ if for every element $a \in$ $X F(S N(a, X)) \subset S N(F(a), Y)$, where $S N(a, X)$ means the smallest neighborhood of $x$ in $X$.

We shall call a map $F: X \rightarrow Y$ ALF continuous at $x \in X$ if $F(S N(x, X)) \subset S N(F(x), Y)$.

In view of Definition 10, we clearly obtain the following properties [9].

(1) Let $f: X \rightarrow Y$ and $g: Y \rightarrow Z$ be continuous maps between ALF spaces. Then the composition $g \circ f: X \rightarrow Z$ is also continuous.

(2) For any element $a \in X$ let $F: X \rightarrow Y$ be a continuous map between ALF spaces. Any elements $x, y \in S N(a, X)$ with $y \in S N(x, X)$ have the following property: $F(y) \in S N(F(x), Y)[9]$.

By using continuous maps between ALF spaces, we obtain the category of ALF spaces which consists of the following two classes:

(1) A class of ALF spaces $(X, T)$;

(2) For every ordered pair of ALF spaces $\left(X, T_{1}\right)$ and $\left(Y, T_{2}\right)$, a class of all ALF continuous maps $f:\left(X, T_{1}\right) \rightarrow\left(Y, T_{2}\right)$ as morphisms.

Since the continuity for maps $f$ between ALF spaces is related to the preservation of the neighborhood relation of the domain of $f$, it is meaningful to characterize the homeomorphism between ALF spaces in such a way:

Definition 11. [9] Let $X$ and $Y$ be two ALF spaces. We say that a map $F: X \rightarrow Y$ is a homeomorphism if $F$ is a continuous bijection and the inverse of $F$ is also continuous.

Example 3.1. In Figure 1 (a), (b) and (c) let us consider the three objects $X, Y$ and $Z$. More precisely, as mentioned at Example 2.1, the set $X$ consists of eight elements, i.e. four faces named by $g_{i}, i \in[0,3]_{\mathbf{Z}}$ and four line segments named by $d_{i}, i \in[0,3]_{\mathbf{Z}}$. The set $Y$ consists of 
twelves elements, i.e. six faces named by $f_{i}, i \in[0,5]_{\mathbf{Z}}$ and six line segments named by $c_{i}, i \in[0,5]_{\mathbf{z}}$. Finally, the set $Z$ also consists of twelves elements, i.e. six faces named by $g_{i}, i \in[0,5]_{\mathbf{Z}}$ and six line segments named by $d_{i}, i \in[0,5]_{\mathbf{z}}$.

Then we can observe that these spaces $X, Y$ and $Z$ are $A L F$ spaces. Let $F: Y \rightarrow Z$ be the map given by $F\left(c_{i}\right)=d_{i}$ and $F\left(f_{i}\right)=g_{i}, i \in[0,5]_{\mathbf{Z}}$. Then $F$ is a homeomorphism.

Note that the same cardinality of $Y$ and $Z$ need not guarantee a homeomorphism. Consider a map $F^{\prime}: Y \rightarrow Z$ such that $F^{\prime}\left(c_{i}\right)=g_{i}$ and $F^{\prime}\left(f_{i}\right)=d_{i}, i \in[0,5]_{\mathbf{Z}}$. While the map $F^{\prime}$ is a bijection, it cannot be continuous.

In addition, we can observe that the two objects $X$ and $Y$ cannot be homeomorphic to each other because their cardinalities are not same.

Remark 3.2. (1) In Figure (a) and (c) consider the four spaces $X, X^{\prime}, Z$ and $Z^{\prime}$. While the spaces $X:=\left\{d_{i}, g_{i} \mid i \in[0,3]_{\mathbf{z}}\right\}$ and $X^{\prime}:=$ $\left\{c_{i}^{0}, c_{i}^{1} \mid i \in[1,4]_{\mathbf{Z}}\right\}$, and $Z:=\left\{d_{i}, g_{i} \mid i \in[0,5] \mathbf{z}\right\}$ and $Z^{\prime}:=\left\{c_{i}^{0}, c_{i}^{1} \mid i \in\right.$ $\left.[1,6]_{\mathbf{Z}}\right\}$ have different shapes, we can consider homeomorphisms between $X$ and $X^{\prime}$ and further, $Z$ and $Z^{\prime}$ as follows: Let $f^{\prime}: X \rightarrow X^{\prime}$ be a map such that $f^{\prime}\left(d_{i}\right)=c_{i+1}^{0}$ and $\left.f^{\prime}\left(g_{i}\right)=c_{i+1}^{1}, i \in[0,3]_{\mathbf{z}}\right\}$ and $h^{\prime}: Z \rightarrow Z^{\prime}$ be a map such that $h^{\prime}\left(d_{i}\right)=c_{i+1}^{0}$ and $\left.h^{\prime}\left(g_{i}\right)=c_{i+1}^{1}, i \in[0,5] \mathbf{z}\right\}$. Then the maps $f^{\prime}$ and $h^{\prime}$ are ALF homeomorphisms.

(2) Compared with a homeomorphism in classical topology (or point set topology including a Hausdorff space), an ALF homeomorphism has its own features. More precisely, while the two objects $X$ and $X^{\prime}$ cannot be homeomorphic to each other from the viewpoint of classical topology, they are homeomorphic from the ALF topological point of view.

\section{Generalization of E.R.Stong's Result related to Finite Spaces}

The paper [17] studied some properties of finite topological spaces related to the classification of homotopy types of finite topological spaces. The author of [17] asserted the following:

Proposition 4.1. [17] Let $F$ be a finite (topological) space, $f$ a continuous map of $F$ into itself. If $f$ is either one-to-one or onto, then it is a homeomorphism.

While the paper [17] proved the assertion in the category of finite topological spaces, we now generalize the above result into that in the category of locally finite topological spaces such as an ALF-category. 
(a)

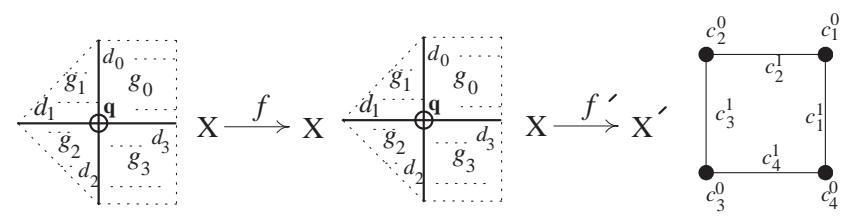

(b)

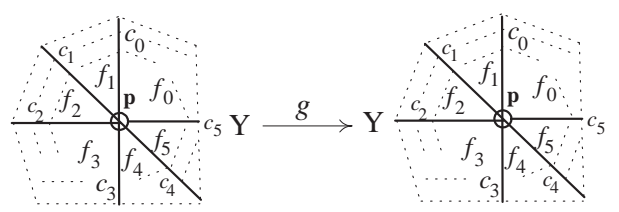

(c)
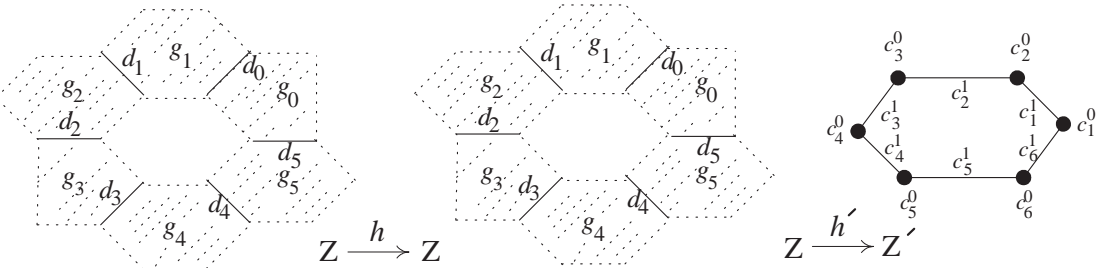

FiguRE 1. Various examples of a homeomorphism in the category of locally finite topological spaces with an ALFstructure.

Since a locally finite space is a generalization of a finite topological space, the following theorem below is a generalized form of Proposition 4.1.

Theorem 4.2. Let $F$ be an ALF space, $f$ a continuous map of $F$ into itself. If $f$ is one to one or onto, then it is a homeomorphism.

Proof: Since $f$ is obviously a continuous bijection, it suffices to prove with the hypothesis that the inverse of the map $f$ is continuous. Consider an arbitrary element $x \in F$, its smallest neighborhood $S N(x, F)$, its image $y=f(x) \in Y$ and the smallest neighborhood $S N(y, F)$. Indeed, $f(S N(x, F))$ is a subset of $S N(y, F)$ and further, owing to the bijection of $f$, the restriction of $f$ on $S N(x, F)$, denoted by $\left.f\right|_{S N(x, F)}: S N(x, F) \rightarrow S N(f(x), F)$, is a homeomorphism. Due to the bijection of $f^{-1}$, we obtain that $f^{-1}(S N(y, F))=S N(x, F)$, which completes the proof.

In digital geometry one of the interesting areas is the Khalimsky $n \mathrm{D}$ space which is a locally finite space and satisfies the separation axiom $T_{0}$ instead of a Hausdorff space if $n \geq 2$. In addition, the Khalimsky 
1D space satisfies the separation axiom $T_{\frac{1}{2}}$ [3]. Thus the present paper mainly studies subspaces of the Khalimsky $n \mathrm{D}$ space from the viewpoint of digital topology.

In relation to the study of discrete objects in $\mathbf{Z}^{n}$, we have used many tools from combinatorial topology, graph theory, Khalimsky topology and so forth $[4,5,6,10,11]$. Motivated by Alexandroff spaces in [1], the Khalimsky $n \mathrm{D}$ space, denoted by $\left(\mathbf{Z}^{n}, T^{n}\right)$, was established and its study includes the papers $[5,9,10,16]$. Since $\left(\mathbf{Z}^{n}, T^{n}\right)$ is the strongest topology induced from the Euclidean $n \mathrm{D}$ topological space, it is useful to consider a subset $X \subset \mathbf{Z}^{n}$ to be a subspace of $\left(\mathbf{Z}^{n}, T^{n}\right)$ denoted by $\left(X, T_{X}^{n}\right), n \geq 1[1,9]$.

In this regard, both a Khalimsky continuous map and a Khalimsky homeomorphism have been often used in digital geometry $[9,10,11,16]$. But it is well known that the graph $k$-connectivity of $\mathbf{Z}^{n}$ and every topological connectedness as well as Khalimsky connectedness are partially compatible with each other [12]. Let us now review some basic notions and properties of Khalimsky $n \mathrm{D}$ spaces. Khalimsky topology can start with the Khalimsky line. More precisely, Khalimsky line topology on $\mathbf{Z}$ is induced from the subbase $\left\{[2 n-1,2 n+1]_{\mathbf{Z}}: n \in \mathbf{Z}\right\}[1]$ (see also [10]). Namely, the family of the subset $\left\{\{2 n+1\},[2 m-1,2 m+1]_{\mathbf{Z}}: m, n \in \mathbf{Z}\right\}$ is a basis of the Khalimsky line topology on $\mathbf{Z}$ denoted by $(\mathbf{Z}, T)$. Indeed, Khalimsky line topology has useful properties. For instance, the Khalimsky line $(\mathbf{Z}, T)$ is connected and if one point is removed, then it consists of two components and is finally not connected [10], which is the similar property of the real line with the usual topology $(\mathbf{R}, U)$, where $\mathbf{R}$ means the set of real numbers. Furthermore, the typical product topology on $\mathbf{Z}^{n}$ induced from $(\mathbf{Z}, T)$, denoted by $\left(\mathbf{Z}^{n}, T^{n}\right)$, is called the Khalimsky $n D$ space. In addition, for two subsets $X$ and $Y$ in $\mathbf{Z}^{n}$ let $\left(X, T_{X}^{n_{0}}\right):=X$ and $\left(Y, T_{Y}^{n_{1}}\right):=Y$ be their Khalimsky subspaces. We recall that a map $h: X \rightarrow Y$ is a Khalimsky (briefly, $K-$ ) homeomorphism if $h$ is a $K$-continuous bijection and further, $h^{-1}: Y \rightarrow X$ is $K$-continuous.

By using the coordinate axis of [11], we can obtain the following:

Lemma 4.3. [13] A Khalimsky topological line space $(\mathbf{Z}, T)$ is equivalent to an $A L F$ space.

Remark 4.4. Consider a self-bijection on the Khalimsky line $(\mathbf{Z}, T)$. Consider the map defined by $f:(\mathbf{Z}, T) \rightarrow(\mathbf{Z}, T)$ with $f(x)=x+$ $2 m, m \in \mathbf{Z}$. Then the map $f$ is a continuous bijection. Further, we can obviously observe that the map $f$ is a homeomorphism. 
Suppose another bijection $g:(\mathbf{Z}, T) \rightarrow(\mathbf{Z}, T)$ with $g(x)=x+2 m+$ $1, m \in \mathbf{Z}$. While the map $g$ is a bijection, it cannot be continuous. Therefore, the map $g$ cannot be a homeomorphism.

Corollary 4.5. Let $(\mathbf{Z}, T)$ be the Khalimsky line. A self-map $f$ : $(\mathbf{Z}, T) \rightarrow(\mathbf{Z}, T)$ is a Khalimsky homeomorphism if $f$ is a continuous map and it is one to one or onto.

Proof: It is well known that the Khalimsky line topology $(\mathbf{Z}, T)$ is an LFS with an Alexandroff topological structure. because $S N(x, \mathbf{Z})=$ $\{x-1, x, x+1\}$ if $x=2 m, m \in \mathbf{Z}$ and $S N(x, \mathbf{Z})=\{x\}$ if $x=2 m+1, m \in$ Z. The proof is completed.

Definition 12. [2] Let $\left(X, \leq_{X}\right)$ and $\left(Y, \leq_{Y}\right)$ be partially ordered sets. We say that a map $F: X \rightarrow Y$ is an isomorphism if $F$ is an order preserving bijection and the inverse of $F$ is also an order preserving map.

Theorem 4.6. Let $F$ be a partially ordered set, $f$ an order preserving map of $F$ into itself. If $f$ is one to one or onto, then it is an isomorphism.

Proof: It is well known that a partially ordered set $(F, \leq)$ induces equivalently an Alexandroff topological space $\left(F, T_{F}\right)$ with $T_{0}$-separation axiom. Namely, every point $x \in F$ has the smallest open neighborhood $S N(x)=\{y \in F \mid x \leq y\}$ for the topology $\left(F, T_{F}\right)$. Besides, an order preserving map between partially ordered sets induces equivalently an continuous map between the induced Alexandroff topological spaces with the $T_{0}$-separation axiom. Thus the induced homeomorphism can be equivalently an isomorphism of $F$ into itself.

Example 4.7. In Figure 2 consider the set $X:=\{a, b, c, d, e, f, g\}$. Assume a binary relation on $X$, denoted by $(X, R)$, as follows: Each element $x \in X$ has a relation with itself. Further, if there is an arrow from $x$ to $y$, then we say that $x \leq y$. Then we can observe that the relation set $(X, \leq)$ is a partially ordered set (briefly, POS). Besides, the element $a$ is the maximal element and the elements $b, c, g$ and $f$ are minimal elements. In this state even though the relation set $(X, \leq)$ has no ALF topological structure, we may only use the process of an establishment of a smallest neighborhood of a given element suggested in Definition 9 so that we can establish a topology on $X$ induced from the above POS by using the smallest neighborhoods of elements in $X$. For instance, for the elements $d$ and $e$ in $X$ we can observe that $S N(d)=$ $\{a, d\}$ and $S N(e)=\{a, d, e\}$, respectively. Namely, a topology on $X$ is determined by the set of smallest neighborhoods of elements of $X$ as 
a base. Then we can obviously observe that this topology is an LFS with an Alexandroff topological structure. By using this topological structure, we can apply the results of Theorem 4.6 into the study of both Alexandroff topological spaces and POSs.

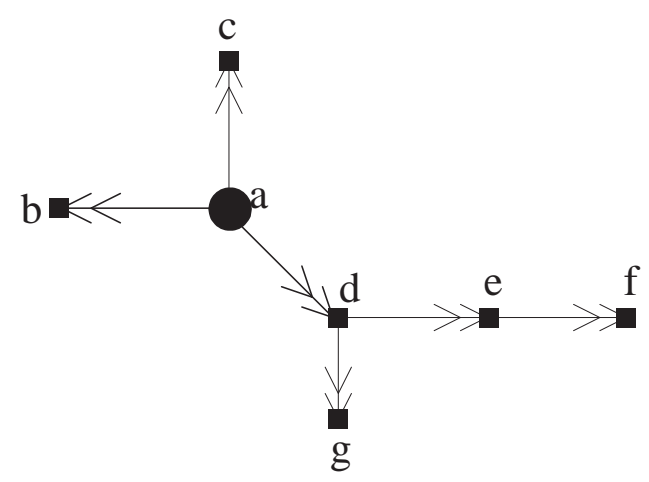

Figure 2. Process on establishing an isomorphism between partially ordered sets or networks.

\section{Summary and Further Work}

We have investigated some properties of ALF spaces which is a special kind of locally finite space. In particular, we have studied some properties of a continuous map and a homeomorphism in the category of ALF spaces. Compared with a general LFS, ALF spaces have strong merits of being easily applicable in computer science and network science. As a further work, we need to study the following:

- New topology for supporting the study of network science.

- New topology for studying LFSs.

- New development of a homotopy for dealing with ALF spaces.

- Algorithm for comparing between ALF spaces up to an ALF homeomorphism.

\section{References}

[1] P. Alexandorff, Diskrete Rume, Mat. Sb. 2 (1937) 501-518.

[2] G. Birkhoff, Lattice Theory, American Mathematical Society, 1961.

[3] W. Dunham, $T_{\frac{1}{2}}$ spaces, Kyungpook Math. J. 17 (1977) 161-169. 
[4] S.E. Han, Strong $k$-deformation retract and its applications, Journal of Korean Mathematical Society 44(6)(2007) 1479-1503.

[5] S.E. Han, Continuities and homeomorphisms in computer topology, Journal of Korean Mathematical Society 45(4)(2008) 923-952.

[6] S.E. Han, Equivalent $\left(k_{0}, k_{1}\right)$-covering and generalized digital lifting, Information Sciences 178(2)(2008) 550-561.

[7] S.E. Han, The $k$-homotopic thinning and a torus-like digital image in $\mathbf{Z}^{n}$, Journal of Mathematical Imaging and Vision 31 (1)(2008) 1-16.

[8] S.E. Han, Extension problem of several continuities in computer topology, Bulletin of Korean Mathematical Society, 47(5)(2010) 915-932.

[9] S.E. Han, Continuity of maps between axiomatic locally finite spaces and its applications, International Journal of Computer Mathematics 88(14) (2011) 28892900.

[10] E. Khalimsky, R. Kopperman, P.R. Meyer, Computer graphics and connected topologies on finite ordered sets, Topology and its Applications 36(1)(1991) 1-17.

[11] V. Kovalevsky, Axiomatic Digital Topology, Journal of Mathematical Imaging and Vision 26 (2006) 41-58.

[12] V. Kovalevsky, Geometry of Locally Finite Spaces, Monograph, Berlin (2008).

[13] V. Kovalevsky, Sang-Eon Han, Product and hereditary property of space set topological structure, Transactions of AMS, submitted.

[14] A. Rosenfeld, Connectivity in digital pictures, Journal of the ACM 17 (1970) 146-160.

[15] H. Seifert and W. Threlfall, A Textbook of Topology, Academic Press, 1980.

[16] J. Stillwell, Classical Topology and Combinatorial Group Theory, Springer (1995).

[17] R.E.Stong, Finite topological spaces, Transactions of AMS 123 (1966) 325-340.

\section{Sang-Eon Han}

Faculty of Liberal Education, Institute of Pure and Applied Mathematics, Chonbuk National University,

Jeonju 561-756, Korea.

E-mail: sehan@jbnu.ac.kr

Chun, Woo-jik

Future Internet Architecture Research Team, Internet Service Research Department, Internet Research Laboratory, ETRI,

Daejeon 305-700, Korea.

E-mail: wjchun@etri.re.kr 